\title{
SIRT6 prevents chondrocyte senescence and DNA damage
}

Previous reports have associated sirtuins, a class of protein deacetylases, with the development of ageing-related diseases. In a new study, Nagai et al. show that inhibition of NAD-dependent protein deacetylase sirtuin-6 (SIRT6) in human chondrocytes is associated with increased DNA damage, telomere dysfunction and premature senescence.

Immunohistochemistry analysis of cartilage tissue from elderly patients with or without osteoarthritis (OA) identified SIRT6-expressing chondrocytes mainly in the superficial zone of articular cartilage, regardless of whether patients had OA or not. In vitro inhibition of SIRT6 expression in chondrocytes with small interfering RNA (siRNA) resulted in increased expression of matrix remodeling proteins $M M P 1$ and $M M P 13$ (fold change $2.3 \pm 0.5$, $P=0.03$ and $4.7 \pm 0.4, P=0.01$, respectively) when compared with chondrocytes transfected with nonsilencing siRNAs. Compared with control cells, chondrocytes incubated with SIRT6 siRNA proliferated less (absorbance $72 \mathrm{~h}$ and $96 \mathrm{~h}$ after treatment $0.77 \pm 0.06$ versus $0.99 \pm 0.06$, $P=0.02$ and $1.16 \pm 0.11$ versus $1.53 \pm 0.10$, $P=0.002$, respectively) and had higher senescence-associated- $\beta$-galactosidase activity (\% positive cells $24.3 \pm 4.2$ versus $11.3 \pm 3.0, P=0.008)$.

To investigate how SIRT6 inhibition led to increased proliferation and senescence in chondrocytes, histone $\mathrm{H} 2 \mathrm{AX}$ phosphorylation $(\gamma \mathrm{H} 2 \mathrm{AX})$ and telomere dysfunction-induced foci (TIF, colocalization of $\gamma \mathrm{H} 2 \mathrm{AX}$ and telomere repeat binding factor-1) were quantified in siRNA-transfected cells to estimate DNA damage and telomere dysfunction. Both the relative area of $\gamma \mathrm{H} 2 \mathrm{AX}$ and the average number of TIFs per cell were higher in chondrocytes treated with SIRT6 siRNA than in control cells $(1.9 \pm 0.1$ versus $1.0 \pm 0.1, P=0.0001$ and $3.5 \pm 0.8$ per cell versus $1.3 \pm 0.2$ per cell, $P=0.007$, respectively), suggesting a role for SIRT6 in DNA repair and telomere homeostasis in these cells. Additionally, the authors found increased levels of cyclin-dependent kinase inhibitor $2 \mathrm{~A}$, isoforms $1 / 2 / 3$ (also known as p16) and decreased levels of cyclindependent kinase inhibitor 1 (also known as p21) in chondrocytes treated with SIRT6 siRNA compared with control cells, implicating SIRT6 in cell-cycle regulation.

These data suggest a role for SIRT6 in preventing DNA damage, telomere dysfunction and premature senescence in chondrocytes-processes that have been implicated in cartilage degeneration in OA.

João H. Duarte

Original article Nagai, K. et al. Depletion of SIRT6 causes cellular senescence, DNA damage, and telomere dysfunction in human chondrocytes. Osteoarthritis Cartilage doi:10.1016/j.joca.2015.03.024 